\title{
Cultural adaptations of CBT for the British Jewish Orthodox community
}

\section{Raphael Kada}

Greater Manchester Mental Health NHS Foundation Trust, Harrop House, Manchester M25 3BL

Received 19 February 2018; Accepted 12 July 2018

\begin{abstract}
There is a national drive to increase access to psychological therapies across England, with a specific focus on under-represented groups such as Black, Asian Minority and Ethnic (BAME) groups. Although prevalence rates for common mental health conditions such as depression and anxiety in Orthodox British Jews are less than those of the generic population in the United Kingdom, accessing services to help treat these conditions within this group are considerably less than other groups. This paper seeks to consider reasons for this, as well as what adaptations, both from a therapist and service perspective, can be made to increase access within the Orthodox Jewish community with lessons to be made to other BAME groups.
\end{abstract}

Key words: CBT, cultural adaptation, religion, community

\section{Introduction}

Improving Access to Psychological Therapies (IAPT) is an English mental health initiative for those with anxiety and depression in primary care, to gain increased and improved treatment as quickly and easily as possible (Roth and Pilling, 2007). This should be accessible to all groups of people within the service area, irrespective of their background (BME Positive Practice Guide - IAPT, 2009).

\section{Why adapt CBT?}

Cognitive behavioural therapy (CBT) has shown in clinical trials to be an effective method of treating common mental health conditions such as anxiety and depression and is currently recommended by National Institute of Clinical Excellence (NICE) guidelines as a first line of treatment for such conditions (NICE, 2009). It is also the treatment most offered to patients presenting within primary care seeking treatment.

While the evidence shows that CBT is effective, this is limited to usual or standard CBT with trials carried out on generic, white and middle class, multi-cultural populations (Hofmann et al., 2012) and so the need to adapt CBT to a target group, especially from Black, Asian Minority and Ethnic (BAME) communities, is necessary. 
Adapting CBT allows for barriers in accessing treatment to be weakened as it enables the provider or practitioner to work towards understanding the particular group they are working with, which in turn encourages greater partnership community working and trust that breaks down stigma. Adapting CBT also allows for CBT to be more accessible to individuals and communities who previously would not have accessed therapy (Guo and Hanley, 2014).

One of the criticisms of CBT is that while it may appeal to the generic population, there are no adaptations made in areas such as formulation or treatment plans (A. Beck and S. Naz, unpublished data), therefore adapting CBT for BAME communities allows for a more unique and specific way of working which leads to better outcomes in therapy (Hinton et al., 2012).

This paper will consider cultural adaptations that might be necessary to meet the needs of the Orthodox Jewish community in the United Kingdom (UK) and which can be potentially carried over into other BAME communities.

This article is a critical reflection (Jarvis, 1992) of the implementation of a project in the accommodation of others that were made in order to develop a bespoke service that catered to the Orthodox Jewish community who wish to maintain their distinct sense of cultural otherness.

Adaptations and ideas mentioned in this article were developed through conversations the author had with key community stakeholders including a respected Rabbi and service users from the community, as well as the managing director of the local IAPT service where this particular Orthodox population resides.

\section{Orthodox Jews}

Jewish identity is changing and often contested, but broadly speaking is grouped into the following categories: (1) Orthodox, (2) Liberal, Conservative and Reform, and (3) nonaffiliated or secular Jews. This article focuses on the first group - the Jewish Orthodox community within the UK who look towards maintaining and upholding the Jewish laws and traditions in what they would describe, in its original manner. This community tends to cluster together geographically usually through the formation of communities to help support each other. As of 2018, the Orthodox populations are mainly gathered around London, Manchester and Newcastle with other smaller communities in areas such as Leeds, Birmingham and Glasgow, enabling the creation of essential Jewish infrastructure such as Synagogues, kosher food, schools, welfare organizations and even health care services such as medical and dental services to take place.

Individuals who describe themselves as Jewish Orthodox are distinct in that they are deeply religious and committed to their faith, yet still maintain a strong allegiance to the host country they live in, working in a diverse range of professions, adhering to British values and blessing the Queen and government in Synagogue on a weekly basis.

The Orthodox Jewish community often have large families with strong family bonds and are a tight-knit community looking to each other for help and support. The Rabbis and elders of the community are highly respected and valued, and are often the first place for those seeking help. They have an unofficial and unspoken, but largely adhered to, dress code consisting of smart, modest and non-eye-catching clothes. This community places great stress on their health and wellbeing, seeing it as a Torah commandment (Deuteronomy - Torah). 


\section{Rates of mental illness and accessing services in this community}

One in six of the general population in the UK experience a mental health condition at one point in their life, with depression and anxiety being the most common (Mental Health Foundation, 2018). Lowenthal et al. (1995) noted a 12\% prevalence rate for depression in Anglo-Jewry and the lower prevalence could be due to strong cohesive family and community bonds, including a multitude of welfare organizations, which can help protect against mental illness. Other elements of the religion such as the weekly day of rest (Sabbath) and their firm belief in the Almighty can also contribute to the communities' wellbeing. While these statistics are over 20 years old, which may make the data redundant, there is little further research evaluating the current prevalence rate for depression in this community. What is known, however, is that the Orthodox population in Stamford Hill, Hackney, a large Jewish population in North London, is rapidly growing with a population figure of 19,781 (7.4\% of Hackney's population) in 2014 rising to an estimated population figure of 26,000 by 2019 (Interlink, Orthodox Jewish Voluntary Action, 2014). Furthermore, there were 2997 recorded referrals received with 63\% reliably improving and 54\% moving to recovery in the 2014 Mental Health Statistics for England (Barker, 2018), indicating that this population group may benefit from IAPT talking therapies. These figures, however, look at the whole Jewish population there, without differentiating between the different Jewish groups.

A local IAPT service within the Greater Manchester area conducted research into the number of individuals seeking therapy from the Manchester Jewish community and found that just two individuals were referred for psychological therapies out of 5548 received in 2011. Clark (2011) expects at least $15 \%$ of the community to be offered treatment, which indicates that Jewish patients are not accessing services.

There is also the need to reflect upon the $84 \%$ increase in anti-Semitic attacks recorded in Greater Manchester over the first 6 months of 2017 (data from Community Security Trust, 2018), that have included arson attacks on two separate Jewish restaurants, amongst others which will no doubt heighten the communities' anxiety over the perceived risk and being the potential target of further attacks. These data have also described $51 \%$ of perpetrators committing anti-Semitic attacks across the UK as 'white European.'

Wallace et al. (2016) found a correlation between increased racism and mental health problems, and as there has been an increase in anti-Semitic attacks across the UK, further up-to-date research would be necessary to understand current prevalence figures for British Jews to consider this relationship.

\section{What are the barriers?}

Research carried out in 2015 within the Manchester Jewish community indicates a high level of concern regarding mental illness. Over $95 \%$ of respondents reported significant levels of stigma and $60 \%$ anticipated fear of using mental health services due to the inaccurate view of social services becoming involved should a parent with mental health conditions be made known to such clinics and the fear of fellow community members becoming aware of their using a mental health service. Additionally, over $60 \%$ of respondents reported concerns that due to their religious needs and requirements, mental health professionals would not understand their situation (Weinberg and Mann, 2015). 
Elements of apprehensiveness, distrust and uncertainty, often present in any mental health setting caused by stigma and not knowing what to expect, may be compounded by a therapist who is from a different culture; this may be compounded by patients who have been on the receiving end of anti-Semitism (Weinberg and Mann, 2015).

There are other barriers too that are found within generic CBT, such as CBT being too goal structured, problem driven, short term and clinical, which will impact those patients wanting to look at exploring their difficulties at a deeper level.

Some ideas around depression only occurring in those who are not truly religious and that the devout Jew should not consult a non-Jewish therapist since it might lead to irreligious ideas or practices (Cinnirella and Lowenthal, 1999) might also present a barrier, and McEvoy et al. (2017) found similar barriers and attitudes in their research with this population.

\section{Adapting for individual therapies}

While it may be standard practice for therapists to greet patients at the entrance and offer them their hand, doing so can be highly uncomfortable to the Jewish patient and can lead to an awkward and unpleasant situation emerging. It also exhibits an unawareness of the community, something hard to have known for the therapist yet basic for the patient. Not only does the Jewish religion prohibit opposite gender touch or contact (with the exception of immediate blood family), it also prohibits seclusion in a locked room with the opposite gender. For CBT therapists, it would be unusual, and perhaps unethical to conduct a therapy session in a locked room, yet an uninformed patient may be unaware that it is on the latch and a simple one-line piece of information informing him or her that the door is closed but not locked can be a reassuring thing to hear.

In adapting therapy to this group, it can be useful to understand that the Rabbi in the community can be a major factor in the success of therapy; should a Rabbi not lend his support to the therapy, the patient is unlikely to be as committed and engaged. Enquiring if the patient's Rabbi is aware he or she is attending therapy, can demonstrate an immediate understanding the therapist has of the community, whilst simultaneously displaying Rogerian core conditions such as care and genuine positive regard. This, however, requires careful consideration and planning to ensure that therapy integrity and fidelity is maintained and upheld as well as ensuring another barrier is not erected in accessing treatment. A CBT practitioner can, however, also meet with the local Rabbi to discuss and explain some background to CBT and identify any spiritual risks or dangers, to allow the CBT therapist to inform their Jewish patients that the talking therapies methodology and techniques to be used have been reviewed and agreed upon by the Rabbi. Doing so may allow for this barrier to be overcome more easily.

Another useful and simple adaption, although perhaps somewhat controversial, is to ask if notes and clinical contacts need to be placed on medical records and if not, to work solely with the patient. It goes without saying that GPs and other agencies will need to be informed should there be risk or harm to self or others and this will need to be made clear at the start of therapy. Excluding these instances, however, this could be something that might not reduce stigma, but increase access to therapy.

Techniques and educative material such as the $\mathrm{ABC}$, five areas or generic formulation models can be adapted to this community to include questions around their culture and religion such as synagogue attendance or Sabbath observance to ascertain their level of functioning as well as the avoidance of questions such as watching television (something 
many Orthodox Jews avoid) and replacing it with culturally specific topics such as their concentration on reading or biblical studies. Furthermore, techniques such as behavioural activation or behaviour experiment exercises require careful planning to ensure that these are culturally appropriate and acceptable to the patient, given that some activities are discouraged within this community. As these are too many to list here, it would be useful to remember that what would be acceptable to a generic population might not be to this patient group. This could be a response to the above-mentioned criticism of CBT formulation driven therapy being independent of the patients' culture and context. Additionally, adding onto the formulation model any instances of anti-Semitism can help the patient feel understood and add uniqueness to the formation of the cycle, allowing the patient to feel heard and valued. Asking about this in a gentle and curious way with some psycho-education offered around how racism can impact ones mental health can allow for their feelings and behaviours and current mental state to be normalized somewhat and puts the therapist in a positive light of someone who respects and understands others.

The use of supervision is a BABCP requirement and is even more important when adapting CBT so that should a therapist feel unconfident in working with an unknown culture, the practitioner can be appropriately supported. Supervisors should also be aware that therapists who see themselves as experienced and skilled in the use of CBT may struggle with those from BAME backgrounds and allowing the supervisee to share his or her vulnerabilities and weaknesses in supervision to allow for the therapist to learn and grow is therefore essential.

\section{Adapting the way services are organized}

Adapting therapy should take place not by setting up separate mental health services but rather changing mainstream services, attitudes and behaviours, without a wider cost to the public (Delivering Race Equality in Mental Health Care, 2009), therefore embedding a new pathway into a current service catering to this client group, would be most appropriate. Although this can be difficult, especially when a community does not engage in accessing generic mainstream services, community engagement where the leaders work in tandem with services to create an appropriate service can increase this dialogue and accessibility into the service (A. Beck and S. Naz, unpublished data). Additionally, services embedded in the community are well suited to meet the needs of older, less affluent patients (Newson et al., 2017). This type of community engagement which Beck and Naz suggest was trialled in 2011 in Salford where the service there noted a year-on-year increase in referrals from the Orthodox Jewish community following extensive dialogue between Rabbinical and communal leaders as well as members of the community coming together to help form a pathway for Orthodox Jews to refer into an already existing service. At present there is also a push for services to be identified and developed by service users with a new initiative entitled 'Patients leading the way in Greater Manchester', which consists of a trial case study on how service users who have utilized services around borderline personality disorder (BPD) come together and feedback their experience alongside professionals, with a view to develop services (McAdam and Heppolette, 2018). The partnership allows for patients to input lived experience alongside the professional viewpoint to look at creating appropriate and timely services. Both these projects engage their respective communities and allow, if nothing else, stigma to be challenged. 
McEvoy et al. (2017) found that through engaging with others in the local community, improved access to health care for under-represented communities such as the Jewish community can begin to occur.

IAPT encourages therapy to be run from satellite locations that are non-stigmatized, neutral places such as fire stations, Sure Start centres and communal venues so that patients receiving treatment do not feel they are attending a mental health clinic or location. This is especially true for communities that are tight knit, such as the Orthodox Jewish community where members socialize, carry out business transactions and intermarry with each other. Setting up clinics in neutral locations can therefore help increase access to those with common mental health conditions, but there is a risk that therapy will not be taken as seriously as patients may associate being treated by a health professional only at a health centre as opposed to a satellite location. Furthermore, some neutral locations may be deemed to be appropriate by the service provider but may be unacceptable to the client. For example, a patient with intrusive obsessive compulsive disorder thoughts regarding children may find attending a Sure Start children's centre hard. Some faith groups may also discourage visiting another religion's place of worship, resulting in therapy being conducted from a religious building as a barrier.

While CBT is traditionally delivered face to face, there is an argument to be made to consider alternative delivery systems which would allow increased accessibility, availability and reduce stigma (Bee et al., 2010). The increased use of online platforms and social media mean that more individuals will have access to these methods where they can access, store, read and re-read material or session content delivered by a CBT therapist. This can then allow for individuals to share some information with family or friends, allowing conversations around mental health to start. This can be especially true for CBT which is educative in nature. To ensure confidentiality is respected and maintained, secure online platforms should be utilized.

As some members of this community are weary about allowing 'outside influences' into their culture, which include books that may contain 'heretical ideas,' the use of some useful self-help material becomes inappropriate and therefore inaccessible. Creating a bibliotherapy as Lovell and Richards (2000) suggest, that sources reliable and appropriate content and approved by the Rabbi, would then allow for this to be shared with patients where necessary.

Lastly, services can seek to employ a diverse and multicultural workforce so that staff can learn from each other which can filter through to patients and also fulfil patient requests to be seen by a particular BAME practitioner if and where appropriate (BME Positive Practice Guide - IAPT, 2009).

\section{Conclusion}

Besides being a government initiative to promote therapy to BAME communities, adaptation of CBT is something that requires minimal adjustment and effort, yet can have farreaching positive consequences on individuals, communities and the wider community. Skills CBT therapists can use include flexibility, creativity and thinking out of the box in BAME communities such as the Orthodox Jewish client group to adapt and promote CBT. At times, a lack of knowledge may prevent therapists from adapting their practice appropriately and having an open and curious attitude allows the therapists to remain interested in patient material (McEvoy et al., 2013) and see, learn and experience others' perspectives. 
Whether it is adapting CBT by offering therapy from a practitioner from within the community, which can be hard at times to source, or offering standard CBT with some minimal adaptations, a better interpersonal rapport will be created, resulting in improved outcomes for patients.

\section{Main points}

(1) There are a number of different sects within the Jewish community.

(2) Barriers are present that prevent the easy access of therapy to the British Jewish Orthodox community.

(3) Adaptations are able to be made that can significantly improve access rates for services and improve the lives of an individual and their communities.

\section{Acknowledgements}

The author would like to acknowledge support given from colleagues who have helped with this paper.

\section{Conflict of interest}

Raphael Kada has no conflict of interest with respect to this publication.

\section{Ethical statement}

The author has abided by the Ethical Principles of Psychologists and Code of Conduct as set out by the APA. No ethical approval was needed.

\section{Financial support}

This research received no specific grant from any funding agency, commercial or not-for-profit sectors.

\section{Recommended further reading}

Community Security Trust (2018). Available at: https://cst.org.uk/data/file/f/c/Antisemitic_ Incidents_Report_Jan-June_2017.1501074748.pdf

Weinberg J, Mann S (2015) Salford Jewish Community Health Research Report, Salford NHS Clinical Commissioning Group.

\section{References}

Barker C (2018). Mental health statistics for England: prevalence, services and funding. House of Commons Library Briefing Paper. Available at: https://researchbriefings.parliament.uk/ ResearchBriefing/Summary/SN06988 
Bee PE, Lovell K, Lidbetter N, Easton K, Gask L (2010). You can't get anything perfect: 'User perspective on the delivery of cognitive behavioural therapy by telephone'. Social Science and Medicine 71, 1308-1315.

Black and Minority Ethnic (BME) Positive Practice Guide - IAPT (2009). Available at: https://www. uea.ac.uk/documents/246046/11919343/black-and-minority-ethnic-bme-positive-practice-guide.pdf (accessed 15 February 2018).

Cinnirella M, Lowenthal KM (1999). Religious and ethnic group influences on beliefs about mental illness: a qualitative interview study British Journal of Medical Psychology 72, 505-524.

Clark DM (2011) Implementing NICE guidelines for the psychological treatment of depression and anxiety disorders: the IAPT experience. International Review of Psychiatry 23, 375-384

Community Security Trust (2018). Available at: https://cst.org.uk/data/file/f/c/Antisemitic_Incidents_ Report_Jan-June_2017.1501074748.pdf (accessed 29 January 2018).

Department of Health (2009). Delivering Race Equality in Mental Health Care - a review. London.

Guo F, Hanley T (2014). Adapting cognitive behavioural therapy to meet the needs of Chinese clients: opportunities and challenges. PsyCh Journal 4, 55-65.

Hinton DE, Rivera EI, Hofmann SG, Barlow DH, Otto MW (2012). Adapting CBT for traumatized refugees and ethnic minority patients: examples from culturally adapted CBT (CA-CBT). Transcultural Psychiatry 49, 340-365.

Hofmann SG, Asnaani AA, Vonk IJJ, Sawyer AT, Fand A (2012). The efficacy of cognitive behavioural therapy: a review of meta-analyses. Cognitive Therapy Research 36, 427-440.

Interlink, Orthodox Jewish Voluntary Action (2014). Community insight report: Patient centred care. Unpublished manuscript.

Jarvis P (1992). Reflective practice and nursing Nursing Education Today 12, 174-181.

Lowenthal K, Goldblatt V, Gorton T, Lubitsch G, Bicknell H, Fellowes D, Sowden A (1995). Gender and depression in Anglo Jewry. Psychological Medicine 25, 1051-1063.

Lovell K, Richards D (2000) Multiple access points and levels of entry (MAPLE): ensuring choice, accessibility and equity for CBT services. Behavioural and Cognitive Psychotherapy 28, 379-391.

McAdam M, Heppolette W (2018). Greater Manchester Borderline Personality Disorder Strategy. Greater Manchester Health and Social Care Partnership, Greater Manchester Combined Authority.

McEvoy P, Baker D, Plant R, Hylton K, Mansell W (2013). Empathic curiosity: resolving goal conflicts that generate emotional distress Journal of Psychiatric and Mental Health Nursing 20, 273278.

McEvoy P, Williamson T, Kada R, Frazer D, Dhliwayo C, Gask L (2017). Improving access to mental health care in an Orthodox Jewish community: a critical reflection upon the accommodation of otherness BMC Health Services Research 17, 557.

Mental Health Foundation (2018). Available at: https://www.mentalhealth.org.uk/statistics/mentalhealth-statistics-uk-and-worldwide (accessed 29 January 2018).

Newson C, Stroebe MS, Schut H, Wilson S, Birrel J, Moerbeek M, Eisma MC (2017). Community-based counselling reaches and helps bereaved people living in low-income households. Psychotherapy Research 1-13.

NICE (2009). Guideline CG90, Depression in adults. Available at: https://www.nice.org.uk/ guidance/cg90

Roth AD, Pilling S (2007). The competences required to deliver effective cognitive and behavioural therapy for people with depression and anxiety disorders. Department of Health, London.

Wallace S, Nazroo J, Bécares L (2016). Cumulative effect of racial discrimination on the mental health of ethnic minorities in the United Kingdom. American Journal of Public Health 106, 1294-1300.

Weinberg J, Mann S (2015). Salford Jewish Community Health Research Report, Salford NHS Clinical Commissioning Group. 


\section{Learning objectives}

(1) To understand diverse cultures and backgrounds, particularly that of the British Jewish Orthodox community.

(2) To consider barriers present in the British Jewish Orthodox community.

(3) To transfer knowledge of the British Jewish Orthodox community to other BAME communities.

(4) To consider adaptations from both a service and therapist perspective to reduce these barriers in accessing and engaging in therapy. 